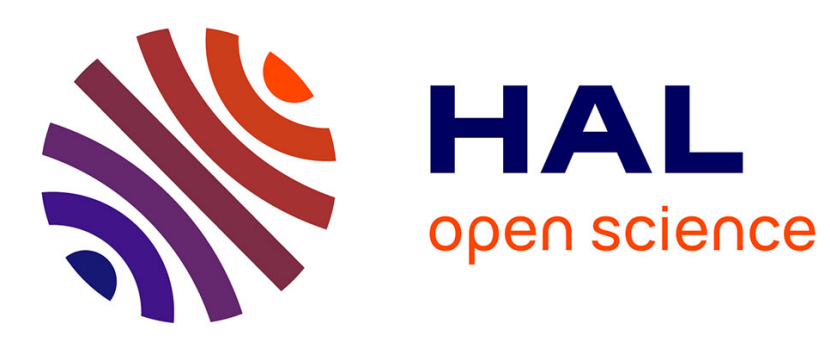

\title{
From Second to Higher Order Tensors in Diffusion-MRI
}

Aurobrata Ghosh, Rachid Deriche

\section{To cite this version:}

Aurobrata Ghosh, Rachid Deriche. From Second to Higher Order Tensors in Diffusion-MRI. AjaFernández, Santiago and de Luis García, Rodrigo and Tao, Dacheng and Li, Xuelong. Tensors in Image Processing and Computer Vision, Springer London, pp.315-334, 2009, Computer Science, 9781-84882-298-6 (Print) 978-1-84882-299-3 (Online). 10.1007/978-1-84882-299-3 . inria-00496858

\section{HAL Id: inria-00496858 https://hal.inria.fr/inria-00496858}

Submitted on 16 Jul 2010

HAL is a multi-disciplinary open access archive for the deposit and dissemination of scientific research documents, whether they are published or not. The documents may come from teaching and research institutions in France or abroad, or from public or private research centers.
L'archive ouverte pluridisciplinaire HAL, est destinée au dépôt et à la diffusion de documents scientifiques de niveau recherche, publiés ou non, émanant des établissements d'enseignement et de recherche français ou étrangers, des laboratoires publics ou privés. 


\title{
From Second to Higher Order Tensors in Diffusion-MRI
}

\author{
Aurobrata Ghosh and Rachid Deriche \\ Project Team Odyssée, INRIA Sophia Antipolis - Méditerranée \\ Aurobrata.Ghosh@sophia.inria.fr
}

Diffusion MRI, which is sensitive to the Brownian motion of molecules, has become today an excellent medical tool for probing the tissue micro-structure of cerebral white matter in vivo and non-invasively. It makes it possible to reconstruct fiber pathways and segment major fiber bundles that reflect the structures in the brain which are not visible to other non-invasive imaging modalities. Since this is possible without operating on the subject, but by integrating partial information from Diffusion Weighted Images into a reconstructed 'complete' image of diffusion, Diffusion MRI opens a whole new domain of image processing. Here we shall explore the role that tensors play in the mathematical model. We shall primarily deal with Cartesian tensors and begin with 2nd order tensors, since these are at the core of Diffusion Tensor Imaging. We shall then explore higher and even ordered symmetric tensors, that can take into account more complex micro-geometries of biological tissues such as axonal crossings in the white matter.

\section{Introduction}

The Brownian motion or diffusion of particles observed by Robert Brown in 1828 was first modelled independently by Albert Einstein in 1905 while trying to provide an experimentally testable hypothesis for the kinetic-molecular theory of matter [12]. Nuclear Magnetic Resonance (NMR) was discovered by Felix Bloch [7] and Edward M. Purcell [32] in 1946. In 1950 Erwin L. Hahn published a paper [16] where he noted that the amplitude of the observed NMR spin echo in the presence of a magnetic field inhomogeneity would be attenuated due to the inherent Brownian motion of the spins. Shortly after, in 1954 Herman Y. Carr and Purcell developed a set of equations [10] to describe this attenuation as a function of discrete motion of the spins. H. C. Torrey subsequently developed the continuum description in 1956 [35]. And about a decade later in 1965 E. O. Stejskal and J. E. Tanner designed the 
classical pulsed gradient spin echo (PGSE) experiment that made it possible to measure the coefficients of molecular diffusion from Diffusion NMR [33].

Magnetic Resonance Imaging (MRI) was developed by Paul C. Lauterbur in 1973 [19] making it possible to generate two and three dimensional images using NMR principles. Peter Mansfield developed the magnetic gradient scheme called Echo Planar Imaging (EPI) in [25]. This facilitated the development of Diffusion MRI (D-MRI), which saw its development in [20, 26, 34].

Since then, D-MRI has come a long way today to become a state-of-the-art medical tool for probing the tissue micro-structure of cerebral white matter in vivo and non-invasively. This became possible due to the concept of Diffusion Tensor Imaging (DTI) introduced in $[5,6]$ and due to the development of the diffusion propagator formalism. DTI and the diffusion propagator formalism provide ways to infer the geometry of the underlying medium.

Here we shall take a look at the role played by Cartesian tensors in the mathematical model of the diffusion propagator and the aquired Diffusion Weighted Images (DWI) or signal. 2nd order diffusion tensors were first introduced by Peter J. Basser in 1994 [5, 6] to accomodate anisotropic diffusion phenomenon in biological tissues, and it became the corner stone in medical D-MRI as DTI. Higher Order and symmetric Tensors (HOT) were recent additions to the propagator and signal model and were introduced to take into account more complex micro-geometries of the underlying tissue such as axonal crossings in the white matter.

We shall begin with the fundamental equations that describe the phenomenon of D-MRI. DTI shall be presented in this framework. DTI with the 2nd order tensor describes Gaussian diffusion or free or unrestricted diffusion. However, the anisotropy that the 2nd order diffusion tensor can describe can only arise from restriction, which would seem to present a contradiction [36]. This shall be resolved by exploring Generalized DTI (GDTI) which will consider the Gaussian model to be a low spatial frequency approximation of the propagator. Two distinct models of GDTI were developed and are often known by the names GDTI-1 and GDTI-2. Both shall be presented, and both employ HOTs to formulate the propagator and signal model. Finally we shall take a look at recent attempts to apply the positivity constraint on the diffusivity function while estimating 2nd and 4th order diffusion tensors from the signal. This is motivated by the fact that diffusion is a positive quantity and negative diffusion has no physical meaning. However, since the DWIs contain noisy signal the estimated tensorial diffusivity functions can often have negative values.

\section{Principles of Diffusion MRI}

We shall begin by examining the fundamental equations governing the principles of D-MRI, namely the Bloch-Torrey equation and the Stejskal-Tanner equation. These will provide the basic framework for the 2nd order diffusion 


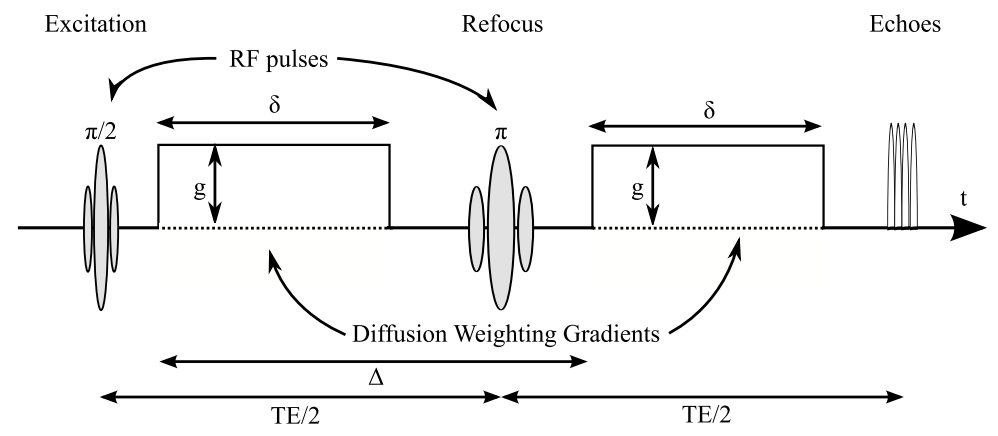

Fig. 1. The PGSE sequence. For the idealised case of rectangular gradient pulses, $g$ represents gradient intensity, $\delta$ gradient duration, and $\Delta$ gradient spacing.

tensor and its role in DTI to describe diffusion anisotropy. We shall then go back to the physics of the problem and present the propagator formulation which will make it possible to describe more complex anisotropies by introducing HOTs in later sections.

\subsection{The Bloch-Torrey Equations}

Torrey proposed the addition of two terms to the Bloch equation to account for flow and diffusion, which gives the Bloch-Torrey equation[35, 9] in the rotating frame and in the absence of radio frequency $(\mathrm{RF})$ field to describe diffusion NMR as

$$
\frac{\partial M_{+}}{\partial t}=-i \gamma \mathbf{r} \cdot \mathbf{g}(t) M_{+}-\frac{M_{+}}{T_{2}}+\nabla \cdot\left(D \nabla M_{+}\right)-\nabla \cdot \mathbf{v} M_{+}
$$

where $M_{+}$is the complex magnetization vector, $\mathbf{r}$ is the spin position vector, $\mathbf{g}(t)$ is the applied magnetic gradient as a function of time, $\gamma$ is the gyromagnetic ratio, $T_{2}$ is the spin-spin or the transverse relaxation time, $\mathbf{v}$ is the flow velocity, $D$ is the diffusion coefficient, and $\nabla .\left(D \nabla M_{+}\right)$is Fick's first law of diffusion. We shall consider the case of pure diffusion where the net flow term $\nabla \cdot v M_{+}$is zero. The following development follows closely $[9,36]$.

The PGSE experiment was designed by Stejskal and Tanner to quantitatively measure the diffusion coefficient (Fig-2.1). Essentially the spin system is excited with a $\pi / 2 \mathrm{RF}$ pulse and a magnetic gradient field constant over time $\delta$ (which is not always achievable in practice) is applied to encode the spin positions. Then the spins are flipped around by a $\pi$ RF pulse and the same time constant magnetic gradient is re-applied after a time $\Delta$. The echo signal is finally aquired after a time TE. If a spin diffuses during the time $\Delta$, then its phase will not return to its original orientation after the second gradient is applied. This increase of disorder in the spin phase distribution 
will cause the spin echo signal to attenuate proportionally to the mean phase difference.

Equation (1) can be solved by realizing that $M_{+}$is a function of both $\mathbf{r}$ and $t$ and by making the substitution

$$
M_{+}(\mathbf{r}, t)=M(t) \exp (-i \mathbf{r} \cdot \mathbf{G}(t)) \exp \left(-t / T_{2}\right)
$$

with

$$
\mathbf{G}(t)=\gamma \int_{0}^{t} \mathbf{g}\left(t^{\prime}\right) d t^{\prime}
$$

in (1), where we now consider $\mathbf{g}$ to be the 'effective gradient' incorporating also the effect of the phase inversion RF pulses. Solving from there for only the real part of $M_{+}(\mathbf{r}, t)$ which corresponds to attenuation due to diffusion, we obtain

$$
M(t)=M(0) \exp \left(-D \int_{0}^{t} \mathbf{G}\left(t^{\prime}\right)^{T} \mathbf{G}\left(t^{\prime}\right) d t^{\prime}\right),
$$

which is the well known Stejskal-Tanner equation [33].

Equation (4), can be rewritten in the following manner by introducing the $b$-factor for $t=T E$

$$
b=\int_{0}^{T E} \mathbf{G}\left(t^{\prime}\right)^{T} \mathbf{G}\left(t^{\prime}\right) d t^{\prime},
$$

and $\mathbf{u}$ a unit vector along the gradient direction $\mathbf{g}$, giving us

$$
M=M(0) \exp \left(-b D \mathbf{u}^{T} \mathbf{u}\right) .
$$

It can be shown that $b=\gamma^{2} g^{2} \delta^{2}\left(\Delta-\frac{\delta}{3}\right)[20,9]$.

Equation (4) can also be modified to introduce the B-matrix which can take into account greater variations in the gradient term to account for imaging and spoiling gradients

$$
\mathbf{B}=\int_{0}^{T E} \mathbf{G}\left(t^{\prime}\right) \mathbf{G}\left(t^{\prime}\right)^{T} d t^{\prime}
$$

then equation (4) becomes

$$
M=M(0) \exp (-D \operatorname{tr}(\mathbf{B})) .
$$

The B-matrix was introduced by Basser [5] and is more generic than the scalar $b$-factor. This can be seen when the diffusion is anistropic and the diffusion coefficient $D$ is replaced by the 2 nd order diffusion tensor $\mathbf{D}$. We will see the 2nd order diffusion tensor shortly. In that case equation (8) becomes

$$
M=M(0) \exp (-\operatorname{tr}(\mathbf{B D})) .
$$

This formulation improves the accuracy by allowing the off-diagonal terms of the $\mathbf{B}$-matrix to couple imaging and diffusion gradient pulses in orthogonal 
directions over and above only the diagonal terms which account for interactions between diffusion and imaging gradient pulses in the same direction [5]. The orthogonal effects are, however, often overlooked, since their strength is typically small in comparison to the diffusion-weighting gradients, and using the $b$-factor $\left(b=B_{11}+B_{22}+B_{33}\right)$ greatly simplifies calculations (reference 44 in $[27])$.

\subsection{Diffusion Tensor Imaging (2nd Order)}

The diffusion phenomenon is called isotropic when the apparent diffusion is independent of the diffusion direction. Anisotropic diffusion occurs when the apparent diffusion varies for different directions. It is generally caused by strongly aligned micro-structures in the medium. Diffusion can therefore occur more freely along the aligned micro-structure, while it is restricted in the perpendicular direction. Anisotropic diffusion provides a convenient way to infer the alignment in the structure of the medium.

To describe Gaussian anisotropy, Basser introduced the 2nd order diffusion tensor, a $3 \times 3$ symmetric positive definite matrix $\mathbf{D}$ to replace the scalar diffusion coefficient $D[5,6]$. It's effect was to modify equation (8) to (9) and (6) to

$$
M=M(0) \exp \left(-b \mathbf{u}^{T} \mathbf{D u}\right) .
$$

The $\mathbf{u}^{T} \mathbf{D u}$ is known as the diffusivity function and is often written as $D(\mathbf{g})=$ $\mathbf{g}^{T} \mathbf{D g}$ with the letter $\mathbf{g}$ replacing the letter $\mathbf{u}$.

DTI provided quantitative framework for describing anisotropic diffusion. It made it possible to identify the alignment of the medium's micro-structure by diagonalizing $\mathbf{D}$ and associating it's major eigenvector to this dominant alignment direction. This in turn made it possible to trace fibers in the brain's white matter.

The six independent coefficients $\left\{D_{i j}\right\}$ of $\mathbf{D}$ and the unweighted MR image $M(0)$ can be estimated from a set of seven or more DWIs. The estimation is linear in its simplest form and can be computed by linearizing equation (10) by taking the logarithms on both sides

$$
\ln (M)=\ln (M(0))-b \mathbf{u}^{T} \mathbf{D u} .
$$

By creating a vector out of the unknowns $\mathbf{d}=\left[D_{11}, D_{12}, D_{13}, D_{22}, D_{23}, D_{33}\right.$, $\ln (M(0))]^{T}$, by computing an $N \times 7$ matrix $\mathbf{Y}$ for the $N$ gradient directions $\{\mathbf{u}\}_{i=1}^{N}$ and $b$-values from (11) such that the $i$-th row is $b_{i} \cdot\left[u_{i 1} u_{i 1}, u_{i 1} u_{i 2}, u_{i 1} u_{i 3}\right.$, $\left.u_{i 2} u_{i 2}, u_{i 2} u_{i 3}, u_{i 3} u_{i 3}, 1 / b_{i}\right]$, and by storing the logarithms of the $N$ observed diffusion signals along the corresponding gradient directions $\left\{\ln \left(M_{i}\right)\right\}_{i=1}^{N}$ in an $N \mathrm{x} 1$ vector $\mathbf{S}$, the unknowns can be estimated as

$$
\mathbf{d}_{\text {opt }}=\operatorname{argmin}_{\mathbf{d}}\|(\mathbf{Y d}-\mathbf{S})\|^{2},
$$

which gives 


$$
\mathbf{d}_{\text {opt }}=\left(\left(\mathbf{Y}^{T} \mathbf{Y}\right)^{-1} \mathbf{Y}^{T}\right) \mathbf{S},
$$

which is the Moore-Penrose pseudoinverse or the linear Least Square (LS) approach.

More sophisticated methods exist [39], many also estimate the diffusion tensor $\mathbf{D}$ in the presence of noisy signal. Basser in [5] takes into account a Gaussian noise model, while other approaches constrain the problem further to guarantee positive diffusivity or to apply spatial smoothing $[11,37,13,28$, $31,22]$.

\subsection{The Diffusion Propagator}

It is important to understand that the signal from the PGSE experiment measures the diffusion of spin bearing particles, and that it is an average of the superimposed signals of a large number of such particles. Therefore to understand and model the signal generation one has to be able to both describe the diffusion motion of a spin bearing particle and also be able to compute something of an 'ensemble average' quatity.

Since the Brownian motion of a particle is erratic and random it is convenient to describe it using a conditional probability density function (PDF) $P\left(\mathbf{r}^{\prime} \mid \mathbf{r}, t\right)$ which is the probability of finding a particle at position $\mathbf{r}^{\prime}$ at time $t$ given that it was at the position $\mathbf{r}$ at time 0 . This PDF or propagator has to then satisfy the diffusion process described by Fick's first law

$$
\mathbf{J}(\mathbf{r})=-D \nabla_{\mathbf{r}} P\left(\mathbf{r}^{\prime} \mid \mathbf{r}, t\right),
$$

with the initial condition

$$
P\left(\mathbf{r}^{\prime} \mid \mathbf{r}, 0\right)=\delta\left(\mathbf{r}-\mathbf{r}^{\prime}\right),
$$

where $\mathbf{J}$ is the particle probability density flux and $D$ is the diffusion coefficient. Conservation of total conditional probability implies the continuity theorem

$$
\nabla_{\mathbf{r}} \mathbf{J}=-\partial P / \partial t
$$

which states that the rate of change of particle displacement probability is equal to the loss of probability due to the particle flux. Combining (14) and (16) gives us the Fick's second law

$$
\partial P / \partial t=D \nabla_{\mathbf{r}} \cdot\left(\nabla_{\mathbf{r}} P\right) .
$$

The differential equation (17) can be solved for unrestricted or free diffusion which has the special boundary condition $P \rightarrow 0$ as $\mathbf{r}^{\prime} \rightarrow \infty$ and with the initial condition (15) [9], yielding

$$
P\left(\mathbf{r}^{\prime} \mid \mathbf{r}, t\right)=(4 \pi D t)^{(-3 / 2)} \exp \left\{-\left(\mathbf{r}-\mathbf{r}^{\prime}\right)^{2} / 4 D t\right\} .
$$


Notice in this equation that $P$ only depends on $\mathbf{r}-\mathbf{r}^{\prime}$ and not on the initial position $\mathbf{r}$.

The Ensemble Average Propagator (EAP) defined as

$$
\bar{P}(\mathbf{R}, t)=\int P(\mathbf{r}+\mathbf{R} \mid \mathbf{r}, t) \rho(\mathbf{r}) d \mathbf{r},
$$

can then be computed, where $\rho(\mathbf{r})$ is the particle density. It is a useful quantity and gives the average probability of any particle to have a displacement $\mathbf{R}$ over time $t$. For the free diffusion propagator (18), since it is independent of the starting position, the EAP is the same for all the particles and can be written as

$$
P(\mathbf{R}, t)=(4 \pi D t)^{(-3 / 2)} \exp \left\{-\mathbf{R}^{2} / 4 D t\right\},
$$

by dropping the overhead bar.

This Gaussian propagator describing free or unrestricted diffusion is defined for isotropic diffusion by the diffusion coefficient $D$. It can be generalized to the case of anisotropic diffusion by introducing the 2nd order diffusion tensor $\mathbf{D}$ in the equation (14) to yield

$$
P(\mathbf{R}, t)=\left(|\mathbf{D}|(4 \pi t)^{3}\right)^{(-1 / 2)} \exp \left\{-\mathbf{R}^{T} \mathbf{D}^{-1} \mathbf{R} / 4 t\right\} .
$$

DTI, therefore, not only provides a framework for describing anisotropic diffusion, for identifying the major micro-structural alignment direction of the medium from the eigen-decomposition of $\mathbf{D}$, but also for connecting the signal (10) to the Gaussian propagator formulation (21) using the tensor D.

The simplicity of the Gaussian or free diffusion both in terms of a model and computational load make DTI today the preferred approach in D-MRI. The decomposition and easy geometric interpretation of the 2nd order tensor $\mathbf{D}$ also plays in its favour. However, one would notice the paradox that though free diffusion is described by a Gaussian EAP, anisotropy can occur only in the presence of restriction. This seeming contradiction is the motivation to move on to a more generic propagator formulation. It will help us understand the Gaussian propagator as a low spatial frequency approximation of the EAP. It will also provide us with a framework to introduce HOTs to be able to describe more complex geometries such as fiber crossings.

\subsection{The Fourier Relationship}

Let us assume that $\delta$ is infinitesimally short, which we shall denote as the short gradient pulse (SGP) condition. For the sake of simplicity let us also assume at first without loss of generality that the gradients are applied along the $\mathrm{x}$ axis and that $g$ denotes only the magnitude of the gradient vector (Fig-2.1) [27]. The dephasing resulting from the two gradient pulses is then 


$$
\begin{aligned}
& \phi_{1}=\gamma \int_{0}^{\delta} g x_{1} d t=\gamma g \delta x_{1}, \\
& \phi_{2}=\gamma \int_{\Delta}^{\Delta+\delta} g x_{2} d t=\gamma g \delta x_{2},
\end{aligned}
$$

where $x_{1}=x(t=0)$ and $x_{2}=x(t=\Delta)$ are the position vectors. The SGP condition allows for $x(t)$ to be constant over the integrals. The sign of $\phi_{1}$ and $\phi_{2}$ are opposite due to the $\pi$ RF pulse. Therefore the total dephasing due to both the gradient pulses of one particle is

$$
\phi=\phi_{2}-\phi_{1}=\gamma g \delta\left(x_{2}-x_{1}\right) .
$$

And so the complex signal from one particle is

$$
c=e^{i \phi}=e^{i \gamma \delta g\left(x_{2}-x_{1}\right)}=e^{i \gamma \delta g X},
$$

where $X$ is the net displacement due to diffusion along the $\mathrm{x}$-axis. In the general case without assumptions on the gradient, which would then have the value $\mathbf{g}$ and the net displacement vector $\mathbf{R}$ the complex signal is given by

$$
c=e^{i \gamma \delta \mathbf{g} \cdot \mathbf{R}} .
$$

To compute the ensemble average complex signal, we have to again take into account that the signal is the superimposed signals from a large number of spin bearing particles whose displacement probability is the EAP $P(\mathbf{R}, t)$, therefore

$$
\begin{aligned}
C(\mathbf{q}, t)=\left\langle e^{i \mathbf{q} \cdot \mathbf{R}}\right\rangle & =\int_{-\infty}^{\infty} e^{i \mathbf{q} \cdot \mathbf{R}} P(\mathbf{R}, t) d \mathbf{R} \\
& =\mathcal{F}[P(\mathbf{R}, t)],
\end{aligned}
$$

where $\mathbf{q}=\gamma \delta \mathbf{g}$, and $\langle\ldots\rangle$ denotes the ensemble average. The ensemble average complex signal is, therefore, the Fourier transform of the EAP. The propagator can therefore be estimated by taking the inverse Fourier transform of the complex signal

$$
P(\mathbf{R}, t)=\mathcal{F}^{-1}[C(\mathbf{q}, t)]
$$

What is interesting, however, is that it can be shown that for a pure diffusion process, the inverse Fourier transform of the complex signal is equal to the inverse Fourier transform of the modulus of the signal[36]

$$
P(\mathbf{R}, t)=\mathcal{F}^{-1}[|C(\mathbf{q}, t)|]=\mathcal{F}^{-1}[S(\mathbf{q}, t)],
$$

which we shall call the modulus Fourier transform. Hence the diffusion signal $C(\mathbf{q}, t)$ is equal to its modulus $C(\mathbf{q}, t)=|C(\mathbf{q}, t)|=S(\mathbf{q}, t)$.

This establishes the Fourier relation between the signal and the EAP. Diffusion Spectrum Imaging (DSI) uses this relation to compute the propagator from the signal [38]. However, it requires aquiring the signal from a detailed sampling of $\mathbf{q}$-space and therefore suffers from lengthy aquisition times. 


\subsection{Cumulant Expansion of the Propagator}

Equation (27) can also be used to interpret the signal as the characteristic function or the complex cumulant generating function of the EAP. This realization permits us to rewrite the logarithm of the signal in function of the cumulants of the EAP. For simplicity of notation we do this for the one dimensional case along, say, the $\mathrm{x}$-axis

$$
\ln (C)=\sum_{n=1}^{\infty} \kappa_{n} \frac{(i \gamma \delta g)^{n}}{n !}
$$

where $\kappa_{n}$ are the cumulants. We shall see in the three dimensional case that these cumulants are HOTs. Assuming a pure diffusion process it can be shown that all odd order cumulants are zero or that the EAP is an even function with respect to the displacement variable. The signal is therefore the modulus signal $S$.

Truncating the cumulant expansion (31) after the second term yields a signal from a Gaussian EAP with $\kappa_{1}=\mu$ the mean and $\kappa_{2}=\sigma^{2}$ the variance. Comparing to the free Gaussian propagator from equation (20) and considering it in the one dimensional case gives $\kappa_{1}=0$ and $\kappa_{2}=2 D t$. This implies that the logarithm of the diffusion signal is

$$
\begin{aligned}
\ln (S)=-\kappa_{2} \frac{(\gamma g \delta)^{2}}{2} & =-\gamma^{2} g^{2} \delta^{2} \Delta D \\
& \approx-b D
\end{aligned}
$$

Equation (33) is essentially equation (6). The SGP condition therefore yields a signal that differs from the finite pulse experiment (33) by $\delta / 3$.

Hence, assuming the SGP condition, the Gaussian propagator or the free diffusion can be viewed as the low spatial frequency approximation of the EAP.

\subsection{Diffusion Kurtosis Imaging}

The cumulant expansion allows us to naturally characterize the deviation of the diffusion from the Gaussian behaviour due to restriction by considering the higher order cumulants [18]. For example truncating the expansion after the fourth order [27] yields

$$
\ln (S)=-\kappa_{2} \frac{(\gamma g \delta)^{2}}{2}+\kappa_{4} \frac{(\gamma g \delta)^{4}}{4 !} .
$$

By defining the excess kurtosis, or the fourth standardized moment as

$$
K=\frac{\kappa_{4}}{\kappa_{2}^{2}}
$$


and again substituting the value of $\kappa_{2}$ from (20) gives

$$
\kappa_{4}=K \kappa_{2}^{2}=4 K D^{2} \Delta^{2},
$$

which therefore yields

$$
\begin{aligned}
\ln (S) & =-b D+\frac{4 K D^{2} \Delta^{2}(\gamma g \delta)^{4}}{4 !} \\
& =-b D+\frac{1}{6} b^{2} D^{2} K .
\end{aligned}
$$

This makes it possible to directly estimate the diffusion kurtosis by taking three DWI measurements for different $b$-values along any gradient direction (including the $b=0$ unweighted image). This approach is, therefore, known as Diffusion Kurtosis Imaging (DKI) [18].

For anisotropic diffusion in three dimensions the diffusion coefficient $D$ is a 2nd order Cartesian tensor $\mathbf{D}$ and the kurtosis coefficient $K$ becomes a 4 th order Cartesian tensor $\mathbf{K}^{(4)}$.

The kurtosis is a measure of the peakedness of the EAP and tells us if the diffusion is more sharply peaked or less sharply peaked than a Gaussian or free diffusion. Gaussian diffusion corresponds to $K=0$. The cumulant expansion has, however, a finite radius of convergence centered around $b=0$, and, therefore, DKI is useful at intermediate and low $b$-values.

\section{Higher Order Tensors in D-MRI}

Tensors in the form of 2nd order diffusion tensors at the core of the DTI framework allow for the inference of the medium's micro-structure analytically and quantitatively. The EAP formulation, however, generalizes the anisotropy model of the diffusion tensor by considering the Gaussian propagator as a low spatial frequency approximation. This is done from the cumulant expansion of the EAP. In DKI we see higher order cumulants being used, especially the 4th order cumulant which is a 4th order Cartesian tensor to examine the deviation of the diffusion from Gaussian or free diffusion.

The EAP formulation offers the possibility of extracting more complex geometric information of the medium's micro-structure, such as fiber crossings, by admitting more general anisotropy models. The anisotropy is often modelled using HOTs, such as in GDTI, which lend greater geometric details to the propagator with their increased multi-linearity. However, it must be noted that the GDTI models are phenomenoligical or that the order of the tensors are increased to fit the diffusion signal data more closely.

At this point it is relevant to mention D-MRI reconstruction schemes that attempt to be model-free or model-independent. The classical approach along this line is DSI. Q-Ball Imaging (QBI) is another [36]. QBI computes a function known as the Orientation Distribution Function (ODF) which is the radial integration of the EAP. No models are set for the propagator however. 


\subsection{Generalizing Fick's Laws}

Generalized Diffusion Tensor Imaging tries to model the diffusion signal containing anisotropies suspected to be more complex than Gaussian anisotropy using higher order tensors. Two GDTI models have been proposed. Both modify Fick's laws of diffusion to accomodate non-Gaussian diffusion.

Let us go back to equations (14) and (17) and rewrite them for $J$ the flux, $D$ the diffusion coefficient, and $C$ the concentration as:

$$
\begin{aligned}
J & =-D \nabla C \\
\frac{\partial C}{\partial t} & =D \nabla^{2} C .
\end{aligned}
$$

And in the case of Gaussian-anisotropic three dimensional diffusion the equations become

$$
\begin{aligned}
J & =-D_{i j} \nabla_{j} C \\
\frac{\partial C}{\partial t} & =\operatorname{tr}\left(D_{i j} \nabla_{i j}^{2} C\right),
\end{aligned}
$$

where $D_{i j}$ are the coefficients of the 2 nd order tensor $\mathbf{D}$. In equations (41) and (42), we use Einstein's notation convention and a repetition of indices, such as $D_{i j} \nabla_{j} C$, implies a summation over the repeated index over all its possible values $\sum_{j} D_{i j} \nabla_{j} C$.

Two generalizations to Fick's laws were proposed independently by the authors in [24] and [30]. They are sometimes referred to as GDTI-1 and GDTI2 respectively [27].

In GDTI-1, Fick's laws in three dimensions are written as an infinite sum of products of tensors of increasing orders with partial derivatives of $C$ of increasing orders

$$
\begin{aligned}
J_{i_{1}} & =-\sum_{k=2}^{\infty}\left[D_{i_{1} i_{2} \ldots i_{k}}^{(k)} \frac{\partial^{(k-1)} C}{\partial x_{i_{2}} \partial x_{i_{3}} \ldots \partial x_{i_{k}}}\right] \\
& =-\sum_{k=2}^{\infty}\left[D_{i_{1} i_{2} \ldots i_{k}}^{(k)} \nabla_{i_{2} \ldots i_{k}}^{(k-1)} C\right] \\
\frac{\partial C}{\partial t} & =\sum_{k=2}^{\infty}\left[D_{i_{1} i_{2} \ldots i_{k}}^{(k)} \nabla_{i_{1} i_{2} \ldots i_{k}}^{(k)} C\right],
\end{aligned}
$$

where $D_{i_{1} i_{2} \ldots i_{k}}^{(k)}$ are the coefficients of the $k$-th order three dimensional Cartesian diffusion tensor $\mathbf{D}^{(k)}$, by an abuse of notation $\nabla_{i_{1} i_{2} \ldots i_{k}}^{(k)} C$ denotes the $k$-th order partial derivatives of $C$, and we again employ Einstein's notation convention of a repetitive index to represent summation. GDTI-1 modifies the fundamental relation between the flux and the concentration in Fick's laws.

In GDTI-2, Fick's laws in three dimensions for spin bearing particles under the influence of a magnetic gradient $\mathbf{g}$ are written for a given order $k$ as 


$$
\begin{aligned}
J & =-D_{i_{1} i_{2} \ldots i_{k}} g_{i_{1}} g_{i_{2}} \ldots g_{i_{k}} \nabla C \\
\frac{\partial C}{\partial t} & =D_{i_{1} i_{2} \ldots i_{k}} g_{i_{1}} g_{i_{2}} \ldots g_{i_{k}} \nabla^{2} C,
\end{aligned}
$$

where $D_{i_{1} i_{2} \ldots i_{k}}$ are the coefficients of $\mathbf{D}^{(k)}, g_{i_{i}} \ldots g_{i_{k}}$ are components of the gradient $\mathbf{g}$, and we use Einstein's notation convention for the indices. GDTI2 modifies Fick's law by replacing the diffusion coefficient by a generalized diffusion function.

\subsection{GDTI-1}

In [24] the author uses the modified Fick's law (44) in the Bloch-Torrey equation (1). Solving this yields the diffusion function

$$
D(\mathbf{g})=\sum_{k=1}^{\infty}\left[i^{2 k} D_{l_{1} l_{2} \ldots l_{2 k}}^{(2 k)} B_{l_{1} l_{2} \ldots l_{2 k}}^{(2 k)}\right],
$$

where $i$ is the imaginary number, $B_{l_{1} l_{2} \ldots l_{k}}^{(k)}$ are the coefficients of the $k$-th order Cartesian tensor $\mathbf{B}^{(k)}$ that is the generalization of the $\mathbf{B}$-matrix (7), and we again use Einstein's summation notation. We only retain the even ordered diffusion tensors here, unlike in the authors presentation [24]. This is justified by the explanation that the even ordered tensors account for the magnitude of the signal while the odd ordered tensors (on the imaginary axis) account for the phase, and as we have seen earlier, for a pure diffusion process the signal is equal to the modulus Fourier transform of the propagator.

What makes GDTI-1 attractive is that with this form of the diffusion function and given the Fourier relation between the propagator and the signal, it is possible to reconstruct the propagator as a function of the estimated higher order diffusion tensors. This can be seen by replacing the diffusion function (47) in the linearized model of the signal, given by taking logarithms on both sides of equation (9), which yields

$$
\ln \left(\frac{M}{M(0)}\right)=\sum_{k=1}^{\infty}\left[i^{2 k} D_{l_{1} l_{2} \ldots l_{2 k}}^{(2 k)} B_{l_{1} l_{2} \ldots l_{2 k}}^{(2 k)}\right] .
$$

The cumulant expansion of the signal (31) written in the three dimensional case using Einstein's summation notation is

$$
\ln (S)=\sum_{j=1}^{\infty}\left[i^{2 j} \frac{K_{l_{1} l_{2} \ldots l_{2 j}}^{(2 j)} q_{l_{1}} q_{l_{2}} \ldots q_{l_{2 j}}}{2 j !}\right],
$$

where only the even ordered cumulants are non-zero, $K_{l_{1} l_{2} \ldots l_{j}}^{(j)}$ are the coefficients of the $j$-th order cumulant $\mathbf{K}^{(j)}$ which is now a $j$-th order Cartesian 
tensor, and $q_{l_{1}} q_{l_{2}} \ldots q_{l_{2 j}}$ are the coefficients of $\mathbf{q}=\gamma \delta g \mathbf{u}$ with $\mathbf{u}$ the unit vector in the direction of the gradient pulse satisfying the SGP condition.

Comparing equations (48) and (49) reveals [24]

$$
\begin{aligned}
K_{l_{1} l_{2} \ldots l_{n}}^{(n)} & =(-1)^{n} n ! D_{l_{1} l_{2} \ldots l_{n}}^{(n)}\left(\Delta-\frac{n-1}{n+1} \delta\right) \\
& \approx(-1)^{n} n ! D_{l_{1} l_{2} \ldots l_{n}}^{(n)} \Delta,
\end{aligned}
$$

or in other words the higher order cumulants $\mathbf{K}^{(n)}$ can be computed from the diffusion tensors of the same order $\mathbf{D}^{(n)}$ that parameterize the generalized Fick's law (46), while the diffusion tensors can be estimated from the signal or DWIs using the linear LS approach [23].

It is then possible to reconstruct the propagator as a function of the cumulants using the Gram-Charlier series [24]

$$
P(\mathbf{r})=N\left(0, K_{l_{1} l_{2}}^{(2)}\right) \times\left(1+\frac{K_{l_{1} l_{2} l_{3} l_{4}}^{(4)}}{4 !} H_{l_{1} l_{2} l_{3} l_{4}}(\mathbf{r})+\ldots\right),
$$

where $N\left(0, K_{l_{1} l_{2}}^{(2)}\right)$ is the normal distribution with zero mean and covariance matrix $K_{l_{1} l_{2}}^{(2)}$, and $H_{l_{1} l_{2} \ldots l_{n}}(\mathbf{r})$ is the $n$-th order Hermite tensor.

If $p_{l_{1} l_{2}}$ be the components of $N\left(0, K_{l_{1} l_{2}}^{(2)}\right)^{-1}$, and if $w_{l_{1}}=p_{l_{1} l_{2}} r_{l_{2}}$, then [24]

$$
H_{l_{1} l_{2} l_{3} l_{4}}(\mathbf{r})=w_{l_{1}} w_{l_{2}} w_{l_{3}} w_{l_{4}}-6 w_{\left(l_{1}\right.} w_{l_{1}} p_{\left.l_{3} l_{4}\right)}+3 p_{\left(l_{1} l_{2}\right.} p_{\left.l_{3} l_{4}\right)},
$$

where indices within parantheses designate that the term is to be averaged over all permutations of those indices that produce different terms, remembering that $p_{l_{1} l_{2}}=p_{l_{2} l_{1}}$ and $w_{l_{1}} w_{l_{2}}=w_{l_{2}} w_{l_{1}}$. This gives the components of the fourth order Hermite tensor for completeness.

\subsection{GDTI-2}

In [30] the author modifies the Bloch-Torrey equation (1) by replacing the diffusion term described by Fick's classical law by the modified diffusion law of equation (46). Solving this modified Bloch-Torrey equation yields the diffusion function of order $k$

$$
D(\mathbf{g})=\sum_{i_{1}=1}^{3} \sum_{i_{2}=1}^{3} \ldots \sum_{i_{k}=1}^{3} D_{i_{1} i_{2} \ldots i_{k}} g_{i_{1}} g_{i_{2}} \ldots g_{i_{k}} .
$$

which can be seen as a extension of the Gaussian diffusion function defined for the 2nd order tensor

$$
D(\mathbf{g})=\mathbf{g}^{T} \mathbf{D g}=\sum_{i=1}^{3} \sum_{j=1}^{3} D_{i j} g_{i} g_{j} .
$$


The $k$-th order diffusion tensor $\mathbf{D}^{(k)}$ has to be of even order and symmetric. The symmetry constraint implies that the coefficients $D_{i_{1} i_{2} \ldots i_{k}}$ are equal under any permutation of the indices, yielding

$$
N(k)=(k+1)(k+2) / 2
$$

independent coefficients. These unknowns can be estimated from the diffusion signal or DWIs in exactly the same fashion as the unknowns of DTI were estimated - by constructing the vectors $\mathbf{d}, \mathbf{S}$ and the matrix $\mathbf{Y}$ appropriately. One can then again use the linear Least Squares approach (13).

The estimated diffusion function $D(\mathrm{~g})$ allows to approximate the diffusion signal, by extrapolating, everywhere in q-space. The EAP has to be, therefore, computed from the diffusion function by extrapolating the diffusion signal and then by numerically computing its inverse Fourier transform (29). This does not give a closed form for the propagator. However, since the diffusion function is more complex, it allows the propagator to model complex anisotropies such as fiber crossings.

\section{Positivity Constraints}

Diffusion is a positive quantity and negative diffusion doesn't correspond to anything physical. This is the reason behind introducing a positive definite 2nd order diffusion tensor by Basser in DTI [6]. However, since the signal is often noisy it is common to estimate non-positive definite tensors using the linear LS approach. Tackling this problem revealed that $3 \times 3$ symmetric positive definite diffusion tensors belong to a Riemannian space, with a Riemannian metric which assigns an inner product to each point of this space. In fact two affine invariant metrics were proposed that rendered the space of symmetric positive definite matrices $S^{+}$complete, allowing various tasks like interpolation and geodesic computation to be naturally confined to this space. These were the Riemannian metric $[13,28,31,22]$ and the Log-Euclidean metric $[2,1]$. The linear LS approach uses the Euclidean metric of $\mathbb{R}^{3 \times 3}$.

It is well known, and as we have seen, DTI, using 2nd order tensors, cannot model complex anisotropic diffusion phenomenon like fiber crossings. It is limited to a single dominant alignment direction (one major eigenvector), and in the case of crossing fibers the tensors become oblate or spherical. GDTI overcomes this shortcoming by estimating the diffusion function with HOTs. However, HOTs in GDTI are also estimated using the linearized LS approach which doesn't guarantee a positive diffusion function. The problem of guaranteeing a positive diffusion using HOTs is rendered hard by the increased multi-linearity of the tensors. Two approaches were proposed recently to tackle 4 th order diffusion tensors in the GDTI-2 model. These shall be presented here. 


\subsection{Riemannian Approach}

In [15] the authors propose to extend the Riemannian framework from 2nd order tensors to the space of 4 th order tensors by mapping a 4 th order $3 \mathrm{D}$ tensor to a 2 nd order $6 \mathrm{D}$ tensor which is a $6 \mathrm{x} 6$ matrix. Then they proceed to use the Riemannian framework for $S^{+}$in the space $S^{+}(6)$ to guarantee a positive diffusion function.

A 4th order tensor is defined to be a linear transformation $\mathbf{A}^{(4)}: \operatorname{Lin}(V) \rightarrow$ $\operatorname{Lin}(V)$, where $V$ is a vector space over $\mathbb{R}^{n}[29]$. The double-dot-product is introduced as $\mathbf{A}^{(4)}: \mathbf{D}^{(2)}=A_{i j k l} D_{k l}$, using Einstein's summation notation, where $\mathbf{D}^{(2)}$ is a 2nd order tensor. Transpose $\mathbf{A}^{(4) T}$ is then defined as $\left\langle\mathbf{A}^{(4)}: \mathbf{D}^{(2)} \mid \mathbf{C}^{(2)}\right\rangle=\left\langle\mathbf{D}^{(2)} \mid \mathbf{A}^{(4) T}: \mathbf{C}^{(2)}\right\rangle$ using the inner-product $\langle. \mid$.$\rangle in$ the space of 2 nd order tensors. And the Euclidean inner-product in the space of 4 th order tensors is defined to be $\left\langle\mathbf{A}^{(4)} \mid \mathbf{B}^{(4)}\right\rangle=\operatorname{tr}\left(\mathbf{A}^{(4) T} \mathbf{B}^{(4)}\right)$.

A 4th order tensor satisfying major and minor symmetries has 21 independent coefficients, in three dimensions, and has an eigen decomposition. If it satisfies total symmetry it has 15 independent coefficients. This symmetry corresponds to the symmetric HOTs in GDTI, and by replacing $k=4$ in (55) one can arrive at the same number of independent coefficients.

A proposition [29] states that

$$
\left\langle\mathbf{A}_{s}^{(4)} \mid \mathbf{B}_{a}^{(4)}\right\rangle=\operatorname{tr}\left(\mathbf{A}_{s}^{(4)} \mathbf{B}_{a}^{(4)}\right)=0,
$$

where $\mathbf{B}_{a}^{(4)}$ is the remainder or anti-symmetric part that remains when the totally symmetric part $\mathbf{B}_{s}^{(4)}$ of a tensor $\mathbf{B}^{(4)}$ is subtracted from itself.

When a 4 th order tensor in three dimensions $\mathbf{A}^{(4,3)}$, satisfies major and minor symmetries it can be mapped to a symmetric 2 nd order tensor in six dimensions $\mathbf{A}^{(2,6)}[4,29]$. The double-dot-product, for a symmetric 2 nd order tensor $\mathbf{D}^{(2,3)}$, can be rewritten as a matrix vector product $\mathbf{A}^{(4,3)}: \mathbf{D}^{(2,3)}=$ $\mathbf{A}^{(2,6)} \mathbf{d}^{(1,6)}$, where $\mathbf{d}^{(1,6)}=\left[D_{11}, D_{22}, D_{33}, \sqrt{2} D_{12}, \sqrt{2} D_{13}, \sqrt{2} D_{23}\right]^{T}$, where $D_{i j}$ are the six independent coefficients of $\mathbf{D}^{(2,3)}$. The diffusion function of GDTI-2 (53) for order 4 can then be written as

$$
\begin{aligned}
D(\mathbf{g}) & =\mathbf{D}^{(2,3)}: \mathbf{A}^{(4,3)}: \mathbf{D}^{(2,3)} \\
& =\operatorname{tr}\left(\mathbf{A}^{(4,3)} \mathbf{G}^{(4,3)}\right),
\end{aligned}
$$

where $\mathbf{D}^{(2,3)}=\mathbf{g} \otimes \mathbf{g}$, with $\mathbf{g}$ the gradient, $\otimes$ the outer-product, and $\mathbf{G}^{(4,3)}=$ $\mathbf{g} \otimes \mathbf{g} \otimes \mathbf{g} \otimes \mathbf{g}$, a totally symmetric 4th order tensor. For computations the equivalent matrix formulation can be used instead

$$
D(\mathbf{g})=\mathbf{d}^{(1,6)^{T}} \mathbf{A}^{(2,6)} \mathbf{d}^{(1,6)} .
$$

The 4 th order diffusion tensor $\mathbf{A}^{(4,3)}$ can be estimated in $S^{+}(6)$ by using the Riemannian metric and an M-estimator $\Psi$, to account for outlier data, along the lines of [21]. The error energy functional that has to be minimized is 


$$
\begin{array}{r}
E\left(\mathbf{A}^{(2,6)}\right)=\sum_{i=1}^{N} \Psi\left(\frac{1}{b_{i}} \ln \left(\frac{M}{M(0)}\right)+\right. \\
\left.\mathbf{d}_{i}^{(1,6) T} \mathbf{A}^{(2,6)} \mathbf{d}_{i}^{(1,6)}\right),
\end{array}
$$

where $N$ is the number of DWIs aquired, as a non-linear gradient descent problem. The gradient of this functional using the Riemannian metric in $S^{+}(6)$ is

$$
\nabla E=\sum_{i=1}^{N} \Psi^{\prime}\left(r_{i}\left(\mathbf{A}^{(2,6)}\right)\right) \mathbf{A}^{(2,6)} \mathbf{d}_{i}^{(1,6)}\left(\mathbf{A}^{(2,6)} \mathbf{d}_{i}^{(1,6)}\right)^{T},
$$

where $r_{i}\left(\mathbf{A}^{(2,6)}\right)=\frac{1}{b_{i}} \ln \left(\frac{M}{M(0)}\right)+\mathbf{d}_{i}^{(1,6) T} \mathbf{A}^{(2,6)} \mathbf{d}_{i}^{(1,6)}$. Since $\mathbf{A}^{(2,6)}$, is estimated in $S^{+}(6)$, the diffusion function (58) is guaranteed to be positive for any $\mathbf{g}$. However, since $\mathbf{A}^{(2,6)}$ is estimated in $S^{+}(6)$, it has 21 independent coefficients, while a 4th order diffusion tensor is totally symmetric and can have only 15. This indeterminacy can be overcome by noticing that $\mathbf{G}^{(4,3)}$ is totally symmetric, therefore

$$
\begin{aligned}
D(\mathbf{g}) & =\operatorname{tr}\left(\mathbf{A}^{(4,3)} \mathbf{G}^{(4,3)}\right) \\
& =\operatorname{tr}\left(\left(\mathbf{A}_{s}^{(4,3)}+\mathbf{A}_{a}^{(4,3)}\right) \mathbf{G}^{(4,3)}\right) \\
& =\operatorname{tr}\left(\left(\mathbf{A}_{s}^{(4,3)} \mathbf{G}^{(4,3)}\right),\right.
\end{aligned}
$$

where the last equality comes from equation (56), $\mathbf{A}_{s}^{(4,3)}$ contains the coefficients of the 4 th order diffusion tensor and $\mathbf{A}_{a}^{(4,3)}$, the residue, contains the excess parameters. The symmetry constraint of $\left\|\mathbf{A}_{a}^{(4,3)}\right\|=0$ can, therefore, be applied by projecting $\mathbf{A}^{(4,3)}$ to its symmetric part $\mathbf{A}_{s}^{(4,3)}$.

\subsection{Ternary Quartics Approach}

The authors in [3] were the first to attempt a positivity constraint on the 4th order diffusion tensor. The diffusion function of GDTI-2 (53) for order 4 was rewritten as

$$
D(\mathbf{g})=\sum_{i+j+k=4} D_{i j k} g_{1}^{i} g_{2}^{j} g_{3}^{k},
$$

due to the bijection that exists between a symmetric tensor of order $k$ and a homogeneous polynomial of degree $k$. In this form, the diffusion function can be seen to be a function of the three variables $g_{1}, g_{2}, g_{3}$, and of total degree four. It is a Ternary Quatic. The positivity constraint on the diffusion function requires the ternary quartic to be positve for any values of the three variables. Hilbert proved in 1888 [17] that

Theorem 1 Every non-negative real ternary quartic form is a sum of three squares of quadratic forms. 
Using this theorem, the diffusion function (62) can be parameterized as

$$
\begin{aligned}
D(\mathbf{g}) & =\left(\mathbf{v}^{T} \mathbf{q}_{1}\right)^{2}+\left(\mathbf{v}^{T} \mathbf{q}_{2}\right)^{2}+\left(\mathbf{v}^{T} \mathbf{q}_{3}\right)^{2} \\
& =\mathbf{v}^{T} \mathbf{Q} \mathbf{Q}^{T} \mathbf{v}=\mathbf{v}^{T} \mathbf{G} \mathbf{v},
\end{aligned}
$$

where $\mathbf{v}$ contains the monomials consisting of the gradient coefficients $\left[g_{1}^{2}, g_{2}^{2}\right.$, $\left.g_{3}^{2}, g_{1} g_{2}, g_{1} g_{3}, g_{2} g_{3}\right]^{T}, \mathbf{Q}=\left[\mathbf{q}_{1}\left|\mathbf{q}_{2}\right| \mathbf{q}_{3}\right]$ is a $6 \times 3$ matrix, and $\mathbf{G}=\mathbf{Q Q}^{T}$ is the 6x6 Gram Matrix which contains 18 independent coefficients, of which 15 are of the 4 th order diffusion tensor. The coefficients of the diffusion tensor can be extracted from $\mathbf{G}$ using a map described in [3], and which closely resembles the map used in $[4,29]$.

Parameterized in this fashion, with $\mathbf{Q}$ estimated from the DWIs, $\mathbf{G}$ computed from $\mathbf{Q}$, to finally extract the coefficients of the 4 th order diffusion tensor from $\mathbf{G}$, ensures that the diffusion function, a ternary quartic, is always non-negative. The energy functional to estimate $\mathbf{Q}$ from $N$ DWIs is

$$
E(\mathbf{Q})=\sum_{i=1}^{N}\left(M_{i}-M(0) e^{-b_{i} \mathbf{v}_{i}^{T} \mathbf{Q} \mathbf{Q}^{T} \mathbf{v}_{i}}\right)^{2} .
$$

For any given $\mathbf{Q}$, however, it is possible to compute a whole family of $\left\{\mathbf{Q}^{\prime}\right\}$ s such that $\mathbf{Q}^{\prime} \mathbf{Q}^{\prime T}=\mathbf{Q Q}^{T}=\mathbf{G}$, from the group of rotation matrices $\mathbf{R}$, since they are orthogonal and, therefore, $\mathbf{R} \mathbf{R}^{T}=\mathbf{I}$, where $\mathbf{I}$ is the identity matrix. This can be seen by constructing $\mathbf{Q}^{\prime}=\mathbf{Q R}$, since $\mathbf{Q}$ is $6 \times 3$ and $\mathbf{R}$ is $3 \times 3$, and computing $\mathbf{Q}^{\prime} \mathbf{Q}^{\prime T}=(\mathbf{Q R})(\mathbf{Q R})^{T}=\mathbf{Q Q}^{T}$. To reduce this infinite solution space to a finite set of solutions, $\mathbf{Q}$ is separated into its upper and lower $3 \times 3$ blocks $\mathbf{A}$ and $\mathbf{B}$ and re-parameterized via a $\mathrm{QR}$-decomposition of its upper block $\mathbf{A}$ as

$$
\mathbf{Q}=\left(\begin{array}{c}
\mathbf{T R} \\
\mathbf{B}
\end{array}\right)=\left(\begin{array}{l}
\mathbf{T} \\
\mathbf{C}
\end{array}\right) \mathbf{R}
$$

where $\mathbf{T R}$ is the $\mathrm{QR}$ decomposition of $\mathbf{A}, \mathbf{T}$ is a lower trangular matrix, $\mathbf{R}$ is an orthogonal matrix, and $\mathbf{C}=\mathbf{B R}^{T}$. Re-parameterized in this fashion $\mathbf{Q}$ now has exactly 15 independent coefficients which corresponds to the number of unknowns of the 4th order diffusion tensor, and $\mathbf{R}$ is simplified when computing $\mathbf{Q Q}^{T}$.

The authors also proposed a distance function between two 4th order diffusion tensors $\mathbf{A}_{1}$ and $\mathbf{A}_{2}$ for spatial regularization. It is defined as

$$
\operatorname{dist}\left(\mathbf{A}_{1}, \mathbf{A}_{2}\right)=\frac{1}{4 \pi} \int_{S^{2}}\left(D_{1}(\mathbf{g})-D_{2}(\mathbf{g})\right)^{2} d \mathbf{g},
$$

where $D_{1}(\mathbf{g})$ and $D_{2}(\mathbf{g})$ are the diffusion functions that correspond to $\mathbf{A}_{1}$ and $\mathbf{A}_{2}$, and the integral is over the unit sphere $S^{2}$. This metric is invariant to rotations and can be computed analytically as a function of the coefficients of the 4 th order difference tensor $\boldsymbol{\Delta}=\mathbf{A}_{1}-\mathbf{A}_{2}$. 


\section{Conclusion}

Since its inception in the mid 1900s, Diffusion MRI has today become a stateof-the-art medical tool for probing cerebral white matter. Its strength lies in being able to infer the micro-structure of the biological tissue non-invasively and in vivo by reconstructing a 'complete' diffusion image by integrating the partial information from DWIs. This is possible due to the anisotropic phenomenon of diffusion in mediums with strongly alligned micro-structures.

Starting from the fundamental equations that describe the diffusion phenomenon, we have presented here the role played by Cartesian tensors in modelling this anisotropic diffusion and from there extracting the geometric information of the underlying tissue. DTI, using 2nd order diffusion tensors was able to model Gaussian-anisotropy and from there extract a single major diffusion direction or structural alignment from its eigen-decomposition. This allowed to trace fibers in the white matter.

However, we saw how the propagator formulation generalized on DTI by considering the Gaussian propagator to be a low order spatial approximation of the actual EAP. It was then possible to model the general EAP by employing higher order Cartesian diffusion tensors which were capable of accounting for more complex anisotropic diffusion phenomena such as fiber crossings.

In the last section we tackled the problem of the positivity constraint on the diffusion function. Diffusion being a physical phenomenon, negative diffusion does not correspond to anything. However, in the presence of noisy data straightforward linear Least Squares approximation can result in a nonpositive diffusion function. This had been tackled by the Riemannian metric formulation in the case of DTI. We saw two recent methods which attempted to tackle this problem in the case of 4 th order diffusion tensors using the GDTI-2 model.

Tensors play an important role in Diffusion MRI. Higher order tensors are a recent addition, but the 2 nd order diffusion tensor in DTI is a corner-stone technique in the clinical scenario today. Recent work in $[8,14]$ have shown how it is possible to use higher order Cartesian tensors to extract the maxima from relevant spherical functions such as the Orientation Distribution Function (ODF) with great precision. Since the maxima of the ODF indicate fiber

directions, extracting them correctly is of fundamental importance in tracing fiber tracts in regions where complex fiber configurations like crossings are possible.

Acknowledgements: We would like to thank Peter Basser, Evren Ozarslan and Maxime Descoteaux for their valuable discussions.

\section{References}

1. V. Arsigny, P. Fillard, X. Pennec, and N. Ayache. Fast and simple calculus on tensors in the log-euclidean framework. In Proceedings of the 8th Int. Conf. on 
Medical Image Computing and Computer-Assisted Intervention, pages 115-122, 2005.

2. Vincent Arsigny, Pierre Fillard, Xavier Pennec, and Nicholas Ayache. LogEuclidean metrics for fast and simple calculus on diffusion tensors. Magnetic Resonance in Medicine, 56(2):411-421, August 2006. PMID: 16788917.

3. Angelos Barmpoutis, Bing Jian, and Baba C. Vemuri. Symmetric positive 4th order tensors \& their estimation from diffusion weighted MRI. In Information Processing in Medical Imaging (IPMI 2007), 2007.

4. Peter J. Basser and Sinisa Pajevic. Spectral decomposition of a 4th-order covariance tensor: Applications to diffusion tensor MRI. Signal Processing, 87:220 236, 2007.

5. P.J. Basser, J. Mattiello, and D. LeBihan. Estimation of the effective selfdiffusion tensor from the NMR spin echo. Journal of Magnetic Resonance, B(103):247-254, 1994.

6. P.J. Basser, J. Mattiello, and D. LeBihan. MR diffusion tensor spectroscopy and imaging. Biophysical Journal, 66(1):259-267, 1994.

7. F. Bloch. Nuclear induction. Physical Review, 70(7-8):460+, October 1946.

8. Luke Bloy and Ragini Verma. On computing the underlying fiber directions from the diffusion orientation distribution function. In $M I C C A I$ (1), pages 1-8, 2008.

9. P. T. Callaghan. Principles of nuclear magnetic resonance microscopy. Oxford University Press, Oxford, 1993.

10. H. Y. Carr and E. M. Purcell. Effects of diffusion on free precession in nuclear magnetic resonance experiments. Physical Review, 94:630-638, May 1954.

11. C. Chefd'hotel, D. Tschumperlé, R. Deriche, and O. Faugeras. Constrained flows on matrix-valued functions : application to diffusion tensor regularization. In Proceedings of ECCV'02, June 2002.

12. A. Einstein. Über die von der molekularkinetischen theorie der wärme geforderte bewegung von in ruhenden flüssigkeiten suspendierten teilchen. Annalen der Physik, 322(8):549-560, 1905.

13. P.T. Fletcher and S. Joshi. Principal geodesic analysis on symmetric spaces: Statistics of diffusion tensors. In Proc. Computer Vision Approaches to Medical Image Analysis, Prague, May 2004.

14. A. Ghosh, E.Tsigaridas, M. Descoteaux, P. Comon, B.Mourrain, and R. Deriche. A polynomial based approach to extract the maxima of an antipodally symmetric spherical function and its application to extract fiber directions from the orientation distribution function in diffusion mri. New York, USA, 10/09/2008 2008.

15. Aurobrata Ghosh, Maxime Descoteaux, and Rachid Deriche. Riemannian framework for estimating symmetric positive definite 4 th order diffusion tensors. In MICCAI (1), pages 858-865, 2008.

16. E. L. Hahn. Spin echoes. Physical Review, 80(4):580+, November 1950.

17. D. Hilbert. uber die darstellung definiter formen als summe von formenquadraten. Mathematische Annalen, 32:342350, 1888.

18. Jens H. Jensen, Joseph A. Helpern, Anita Ramani, Hanzhang Lu, and Kyle Kaczynski. Diffusional kurtosis imaging: The quantification of non-gaussian water diffusion by means of magnetic resonance imaging. Magnetic Resonance in Medicine, 53:1432-1440, 2005.

19. P.C. Lauterbur. Image formation by induced local interactions: examples employing nuclear magnetic resonance. Nature, 242:190-191, 1973. 
20. D LeBihan and E. Breton. Imagerie de diffusion in vivo par résonnance magnétique nucléaire. CR Académie des Sciences, (301):1109-1112, 1985.

21. C. Lenglet, M. Rousson, R. Deriche, and O. Faugeras. Statistics on the manifold of multivariate normal distributions: Theory and application to diffusion tensor MRI processing. Journal of Mathematical Imaging and Vision, 25(3):423-444, 2006.

22. Christophe Lenglet. Geometric and Variational Methods for Diffusion Tensor MRI Processing. PhD thesis, Universite de Nice - Sophia Antipolis, 2006.

23. C. Liu, R. Bammer, B. Acar, and M. E. Moseley. Characterizing nongaussian diffusion by using generalized diffusion tensors. Magnetic Resonance in Medicine, 51:924-937, 2004.

24. Chunlei Liu, Roland Bammer, and Michael E. Moseley. Generalized diffusion tensor imaging (GDTI): A method for characterizing and imaging diffusion anisotropy caused by non-gaussian diffusion. Israel Journal of Chemistry, 43:145-154, 2003.

25. P. Mansfield. Multi-planar image formation using nmr spin echoes. Journal of Physics C, 10:55-58, 1977.

26. K.D. Merboldt, W. Hanicke, and J. Frahm. Self-diffusion nmr imaging using stimulated echoes. J. Magn. Reson., 64:479-486, 1985.

27. Ludovico Minati and Wladyslaw P. Weglarz. Physical foundations, models, and methods of diffusion magnetic resonance imaging of the brain: A review. Concepts in Magnetic Resonance Part A, 30A(5):278-307, 2007.

28. M. Moakher. A differential geometric approach to the geometric mean of symmetric positive-definite matrices. SIAM J. Matrix Anal. Appl., 26(3):735-747, April 2005.

29. Maher Moakher. The algebra and geometry of fourth-order tensors with application to diffusion MRI. In Joachim Weickert and Hans Hagen, editors, Perspectives Workshop: Visualization and Image Processing of Tensor Fields, number 04172, 2006.

30. Evren Ozarslan and Thomas H Mareci. Generalized diffusion tensor imaging and analytical relationships between diffusion tensor imaging and high angular resolution diffusion imaging. Magn Reson Med, 50(5):955-965, Nov 2003.

31. X. Pennec, P. Fillard, and N. Ayache. A riemannian framework for tensor computing. Technical Report 5255, INRIA, Sophia Antipolis, July 2004.

32. E. M. Purcell, H. C. Torrey, and R. V. Pound. Resonance absorption by nuclear magnetic moments in a solid. Physical Review, 69(1-2):37+, January 1946.

33. E. O. Stejskal and J. E. Tanner. Spin diffusion measurements: Spin echoes in the presence of a time-dependent field gradient. The Journal of Chemical Physics, 42(1):288-292, 1965

34. D. G. Taylor and M. C. Bushell. The spatial mapping of translational diffusion coefficients by the nmr imaging technique. Physics in Medicine and Biology, 30(4):345-349, 1985.

35. H. C. Torrey. Bloch equations with diffusion terms. Phys. Rev., 104(3):563-565, Nov 1956.

36. D.S. Tuch. Diffusion MRI of complex tissue structure. PhD thesis, Division of Health Sciences and Technology, Massachusetts Institute of Technoloy, 2002.

37. Z. Wang, B.C. Vemuri, Y. Chen, and T.H. Mareci. A constrained variational principle for direct estimation and smoothing of the diffusion tensor field from complex DWI. IEEE Transactions on Medical Imaging, 23(8):930-939, 2004. 
38. V. J. Wedeen, T. G. Reese, and D.S. et al. Tuch. Mapping fiber orientation spectra in cerebral white matter with fourier-transform diffusion mr. [abstract] 8th International Society for Magnetic Resonance in Medicine, page 82, 2000.

39. C.F. Westin, S.E. Maier, H. Mamata, A. Nabavi, F.A. Jolesz, and R. Kikinis. Processing and visualization for diffusion tensor mri. Medical Image Analysis, 6(2):93-108, June 2002. 